ACCEPTED MANUSCRIPT

\title{
An alternative approach to evidence the structural conditioning in the dynamic slowdown in a polymer glass-former
}

To cite this article before publication: Cristian Balbuena et al 2019 J. Phys.: Condens. Matter in press https://doi.org/10.1088/1361-648X/ab4a67

\section{Manuscript version: Accepted Manuscript}

Accepted Manuscript is "the version of the article accepted for publication including all changes made as a result of the peer review process, and which may also include the addition to the article by IOP Publishing of a header, an article ID, a cover sheet and/or an 'Accepted

Manuscript' watermark, but excluding any other editing, typesetting or other changes made by IOP Publishing and/or its licensors"

This Accepted Manuscript is @ 2019 IOP Publishing Ltd.

During the embargo period (the 12 month period from the publication of the Version of Record of this article), the Accepted Manuscript is fully protected by copyright and cannot be reused or reposted elsewhere.

As the Version of Record of this article is going to be / has been published on a subscription basis, this Accepted Manuscript is available for reuse under a CC BY-NC-ND 3.0 licence after the 12 month embargo period.

After the embargo period, everyone is permitted to use copy and redistribute this article for non-commercial purposes only, provided that they adhere to all the terms of the licence https://creativecommons.org/licences/by-nc-nd/3.0

Although reasonable endeavours have been taken to obtain all necessary permissions from third parties to include their copyrighted content within this article, their full citation and copyright line may not be present in this Accepted Manuscript version. Before using any content from this article, please refer to the Version of Record on IOPscience once published for full citation and copyright details, as permissions will likely be required. All third party content is fully copyright protected, unless specifically stated otherwise in the figure caption in the Version of Record.

View the article online for updates and enhancements. 


\title{
An alternative approach to evidence the structural conditioning in the dynamic slowdown in a polymer glass-former
}

\section{Cristian Balbuena}

E-mail: cbalbuena@fi.mdp.edu.ar

Institute of Materials Science and Technology (INTEMA), University of Mar del Plata and National Research Council (CONICET), J. B. Justo 4302, 7600 Mar del Plata, Argentina

\section{Ezequiel R. Soulé}

E-mail: ersoule@fi.mdp.edu.ar Institute of Materials Science and Technology (INTEMA), University of Mar del Plata and National Research Council (CONICET), J. B. Justo 4302, 7600 Mar del Plata, Argentina

\begin{abstract}
.
Dynamic slowdown of liquids, leading to a breakdown of Arrhenius behavior of relaxation and Stokes-Einstein relationship (SER), as the glass transition is approached, is still not fully understood despite decades of study. They are usually associated to the emergence of dynamic heterogeneity, that is, regions or clusters of particles that have high or low mobilities. But the physical origin of these dynamic heterogeneity, and in particular, the question whether they have a structural origin or they are a purely dynamical phenomenon, is still under debate. In this work we study through Molecular Dynamics simulations in a polymer model the dynamic slowdown and the breakdown of SER, in connection with dynamic susceptibility calculated for an isoconfigurational ensemble, such that the effects of structure on dynamics can be discriminated. The onset of structure effects on dynamical behavior is found to be coincident with the onset of slow dynamics and SER breakdown.
\end{abstract}

Submitted to: J. Phys.: Condens. Matter 


\section{Introduction}

Glasses are deeply-supercooled solid-like amorphous materials; mechanically they behave as solids, but in their molecular structure they look like liquids. Diverse viewpoints have been developed to understand the dynamics of glass-forming fluids [1,2]. Understanding how the solidity that arises in the material, in any relevant time scale, involves a small, even negligible apparent change in structure, has proven to be an important challenge. The relationship between structure and dynamics in supercooled liquids is a key problem in the physics of glassy materials and a comparison between the behavior of static and dynamic length scales is expected to provide valuable insights in understanding this phenomenology $[3,4,5]$.

The dramatic slowdown of dynamics upon cooling in glass-forming liquids is described typically by the temperature dependence of transport coefficients, shear viscosity $(\nu)$ and the translational diffusion coefficient $(D)$, with the relaxation time scales like the structural relaxation time $\left(\tau_{\alpha}\right)$ obtained from the long-time decay of density correlation functions. The Stokes-Einstein relation (SER) describes how the diffusion constant and the structural relaxation time are related, stating that $D \propto T \tau^{-1}$. As temperature $\mathrm{T}$ of the glass-former decreases, the liquid becomes more and more viscous, and as it approaches glass transition the increase in relaxation time overtakes the decrease in diffusion, leading to the breakdown of SER. It has been empirically found that, when this relationship is lost, a fractional SER holds for a wide range of liquids, such as molecular liquids and atomic liquids. The breakdown of SER has been considered to be one of the hallmarks of glassy dynamics in liquids. Despite many years of detailed study, temperature dependence of transport coefficients and structural relaxation times still remains a hot topic of debate. While some studies have shown that the breakdown in the relation appear in the vicinity of the glass transition temperature [6], there are other studies suggesting its occurrence at a temperature much higher than the mode-coupling temperature [7] and at temperâtures even higher than the melting temperature [8]. As of yet, there is no agreement on the nature and origin of the breakdown of SER. The question whether it has a structural origin or not remains inconclusive. A commonly proposed explanation is the presence of dynamic heterogeneity, specifically the presence of particles having excessively high and low mobilities relative to ideal Brownian motion $[9,10,11,7,12]$.

It is known that when a glass former approaches $T_{g}$ the dynamics becomes more heterogeneous; that is, regions of high and low mobility appear in the system. These regions of similar mobilities are usually associated with the cooperative relaxation regions of the Adam-Gibbs theory [13], which describes the increase in viscosity or relaxation times in terms of thermodynamic factors. Another, more robust, thermodynamic theory of glass transition is Random First-Order Transition (RFOT) [14], which describes the material in terms of a "mosaic" structure of regions with subtle, maybe undetectable, structural differences, that lead to dynamic heterogeneity. Both theories consider that the slowdown in the dynamic is due to thermodynamic aspects, and hence the many efforts trying to find a structural correlation length that can be connected to dynamic behavior. The use of Point-to-Set, an agnostic method, has been successful for reinforcing the validity of the aforementioned theories [15]. Complementarily, great effort was made in recent years to characterize and relate a region of low or high mobility with some specific structural parameter $[16,17,18,19]$. In some systems there has been success in characterizing certain types of local/structures that lead to the particles having low mobility [20, 3]. In a more universal vision about the glass transition, these results are not very encouraging because these structures are system dependent [21]. In a dynamic approach it has become possible to define and measure observables, the fourpoint correlation functions, that can be determined without arbitrariness in a range of systems and may sometimes be inferred from experimental data. They measure a dynamic length scale that can be associated with the increase of dynamic heterogeneity, and are now broadly accepted as standard tools for analyzing dynamical heterogeneity [22, 23, 24]. Simulation studies and recent experiments indicate that an increasing susceptibility $\chi_{4}$ is directly linked to clustering of mobile particles, and it measures and characterizes the size of growing cooperative motion or dynamically correlated mesoscopic domains $[25,26]$.

One of the problems that arise when analyzing the supercooled state of glass-former is that the structural correlation lengths show a much smaller increase with temperature than the dynamic lengths, where the latter increases more in the temporal scales of the 
simulation $[27,4,5]$. This discrepancy found between the different correlation lengths is still a subject of debate and it is a key point since it can reveal if the glass transition contains a thermodynamic origin [28, 29, 30, 31, 32, 33, 34, 5].

A method used in Molecular Dynamics simulations and that has proved to be very effective in demonstrating heterogeneous dynamics, and can aid in discriminating structural from purely dynamic effects, is the Isoconfigurational Ensemble Method (ICEM) $[35,36]$. This method consist in performing many simulations where the particles have different initial velocities, but the same positions. It has been observed that the propensity, which is the ICE average of displacement of a single particle, is not the same for every particle but has a distribution, and both particles with high propensity, and low propensity, tend to form clusters. Although a relation between particle propensity and clustering with the local structure has not been found, a correlation was observed with localized soft modes [37]. In previous works, we have have found, using ICEM, regions where the particles are strongly dynamically connected, as defined by a high value of Pearson's correlation coefficient; these particles also form clusters, but they are not the same than those formed by mobile or immobile particles [38]. In addition, we showed that a dynamic correlation length, defined from the decay of Pearson's correlation coefficient, shows a linear relation with a static correlation length in the whole temperature range, unlike typical four-point correlation lengths which display this linearity only for high temperatures [39].

In this work we study dynamical and structural behavior of a polymer glass-former model in a wide range of temperatures in the supercooled regime. We analyzed the breakdown of SER that emerges at intermediate temperature and its relation with structural aspects and with the heterogeneous dynamics. We find a temperature above $T_{g}$ in which structure plays a determining role in the dynamic heterogeneity and also in the breakdown of SER. These results provide important insights for understanding the dynamic properties in glass-forming liquids.

\section{Methods}

Molecular Dynamic simulations in a polymer glassforming system were performed in a wide range of temperatures and structural relaxation time. The polymer was represented through the bead-spring model with fully flexible chain molecules [40]. Our results are primarily based on simulations containing 400 chains polymers, each chain consisting of 30 monomers. At this length, the chains are unentangled. In some cases, system with 2000 chains were analyzed in order to improve the statistics. All monomers interact through the Lennard-Jones potential:

$U\left(r_{i j}\right)=4 \epsilon\left[\left(\frac{\sigma}{r_{i j}}\right)^{12}-\left(\frac{\sigma}{r_{i j}}\right)^{6}\right]$,

where $r_{i j}$ is the distance between the beads $i$ and $j, \sigma$ is the distance at which the interparticle potential is zero, and $\epsilon$ is the depth of the potential at the minimum. These parameters are used as length and energy units and are given a value of $1 . U\left(r_{i j}\right)$ is truncated for $r_{i j}$ greater than $2.0 \sigma$ with the long-tail correction applied. In addition, the bonded neighbors in a chain interact through the FENE bond potential:

$U_{\text {bond }}\left(r_{i j}\right)=-15 R_{0}^{2} \ln \left[1-\left(r_{i j} / R_{0}\right)^{2}\right]$,

where $R_{0}=1.5 \sigma$ is the maximum length of the bond. All values we report are in standard reduced LJ units.

Simulations were carried out using LAMMPS molecular dynamics software [41]. The Nosé-Hoover thermostat and/Andersen barostat were used to control the temperature $\mathrm{T}$ and the pressure $\mathrm{P}$ of the system, respectively. For all the simulations in this study, the pressure of the system was set to zero $(\mathrm{P}=0)$. Each simulation consisted of a total number of 3990 particles contained in a box with periodic boundary conditions. The NVE ensemble was used for production runs and for latter production of the ICEM. The latter consisted of 500 trajectories and the initial configuration derived from a full equilibrated trajectory, in which we employ NPT and NVT ensemble for the equilibration protocol. This was done at each temperature studied. The absence of drift in thermodynamic magnitudes and the absence of aging was verified for every temperature. The details of the equilibration protocol are in our previous work [38]. For $T>0.50$, every system under study can be equilibrated within a reasonable computation time. The $T_{g}$ of this model, measured as the temperature at which a change in slope of the specific volume as a function of temperature is observed, is close to 0.45 .

\section{Results and discussion}

The relaxation of this model have been extensively studied in previous simulations [40, 42, 43, 44, 45, 46]. The structural relaxation time $\tau_{\alpha}$ can be defined as the time at which the incoherent intermediate scattering function $F_{s}\left(q, \tau_{\alpha}\right)=e^{-1}$. Figure 1 shows the relaxation times as a function of the inverse temperature. The behavior of $\tau_{\alpha}(T)$ is well described by Vogel-Fulcher-Tamman (VFT) expression: $\tau_{\alpha}(T)=$ $\tau_{0} \exp \left(A /\left(T-T_{0}\right)\right)$. It can be noted from this figure that at high temperatures the behavior is Arrhenius type, with a single energy for the relaxation process. Below a certain temperature, the system presents a 
super-Arrhenius behavior and from small temperature changes, drastic changes occur in $\tau_{\alpha}$. A change from Arrhenius to super-Arrhenius temperature dependence of relaxation times defines the onset temperature of slow dynamics, defined as $T_{A}$. In this sense, $T_{A}$ is presented as a reference temperature, which marks the change in behavior that occurs in the relaxation of the system. As can be seen in Figure 1 through the black dashed line, for this model $T_{A}$ is about 0.7-0.75. An alternative way of visualizing this temperature is by observing the behavior of the Mean Square Displacement at the different temperetatures. For temperatures below $T_{A}$, this magnitude shows the appearance of a plateau (cage regime) at intermediate times, larger than the ballistic regime, and smaller than diffusive time. Alternatively, by observing the behavior of incoherent intermediate scattering function, two relaxation mechanisms can be observed for temperatures below $T_{A}$ : the $\beta$ at short times and the alpha at long times. From our previous work [39] it can be noted that both functions manifest the change in behaviour described above at a temperature close to $T_{A}$.

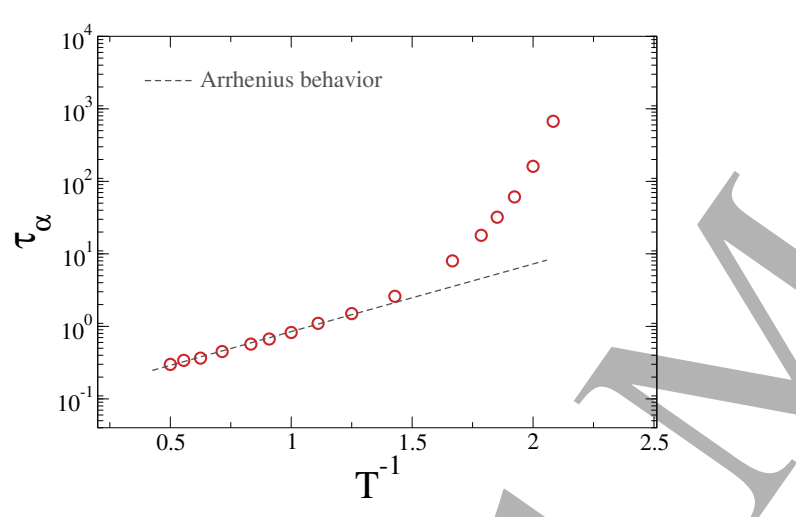

Figure 1. Behavior of structural relaxation times as a function of temperature. The dashed line highlights the behavior of the Arrhenius type that is given for $T>T_{A}$, where for $T<T_{A}$ the linearity is lost, typical phenomenology of fragile glasses.

In what follows, we characterized the heterogeneous dynamics by two functions that are typically used to describe it. On the one hand we use the dynamical susceptibility $\chi_{4}(t)$, which is the integral of the four-point correlation function $g_{4}(r, t)$. We calculate the dynamic susceptibility $\chi_{4}(t)$ from the immobile particles in the system through the fluctuation of an overlap function $Q(t)$ defined us:

$Q(t)=\sum_{i j}^{N} w\left(\left|r_{i}(0)-r_{j}(t)\right|\right)$,

where $w\left(\left|r_{i}(0)-r_{j}(t)\right|\right)$ is a window function that is defined to be unity if $\left|r_{i}(0)-r_{j}(t)\right|<a, 0$ otherwise, where $a=0.3$. So the $Q(t)$ measures the number of overlapping particles in two configurations separated by a time interval $t$ [23]. The fluctuation in this magnitude is linked to the degree of dynamic heterogeneity[23] and $\chi_{4}(t)$ is measured using the expression:

$\chi_{4}(t)=\frac{\beta V}{N^{2}}\left[<Q(t)^{2}>-<Q(t)>^{2}\right]$.

For supercooled liquids, $\chi_{4}(t)$ shows a peak at a time proportional to the $\alpha$ relaxation time or $\tau_{\alpha}$. Figure 2(a) shows the behavior of this function, where it can be seen that, as temperature decreases, this magnitude grows. In addition, the time at which the maximum in $\chi_{4}(t)$ occurs also increases with a decrease in temperature. This time is defined/as $t_{4}$. On the other hand, we employed the non-Gaussian parameter $\alpha_{2}(t)$ defined as:

$\alpha_{2}=\frac{3\left\langle r^{4}(t)\right\rangle}{5\left\langle r^{2}(t)\right\rangle^{2}}-1$

which quantifies how the distribution of particle displacements in time $t$ deviates from Gaussian form, expected for spatially homogeneous dynamics. The non-Gaussian parameter, calculated from the second and fourth moments of displacement distribution, exhibits a peak at a characteristic time $t^{*}$ that increases as the temperature is decreased. Figure 2 (b) shows the non-Gaussian parameter for different temperatures. The same behavior is observed in every case: at small times, ballistic movement, which has a Gaussian distribution, is observed and $\alpha_{2}(t)$ is zero. For intermediate times, $\alpha_{2}(t)$ rises reaching a maximum at $t^{*}$. Eventually, at long enough times monomer movement reaches a diffusive regime and $\alpha_{2}(t)$ decreases approaching zero again. From Figure $2(\mathrm{a})$ and (b) it can be noticed that both magnitudes, $\chi_{4}(t)$ and $\alpha_{2}(t)$, have a similar behavior in that they approach zero at short and long times and they have a maximum. Nevertheless, the position of the curves in the temporal scale is not the same. This can be better seen in Figure 2(c), where the relative behavior of both magnitudes at three temperatures is shown, highlighting the temporal position of their maxima, $t_{4}$ and $t^{*}$. At high temperatures, both characteristic times are coincident, but they decouple at low $\mathrm{T}$.

Figure $3 \mathrm{a}$ shows the behavior of $t^{*}$ and $t_{4}$, compared to $\tau_{\alpha}$, in the whole temperature range studied. At high temperatures the three characteristic times are equal but, for low temperatures $t^{*}$ deviate from $\tau_{\alpha}$ and $\chi_{4}(t)$. In contrast, the value of $t_{4}$ is similar to $\tau_{\alpha}$ in the whole temperature range. These characteristic times can be associated with extremes in mobility behavior, as described in reference [46]. In that workit was shown that $t^{*}$ scales linearly with the reduced diffusion coefficient $D / T$. In this manner, the time scale of mobile particles relate to a diffusive 
An alternative approach to evidence the structural conditioning in the dynamic slowdown in a polymer glass-former5

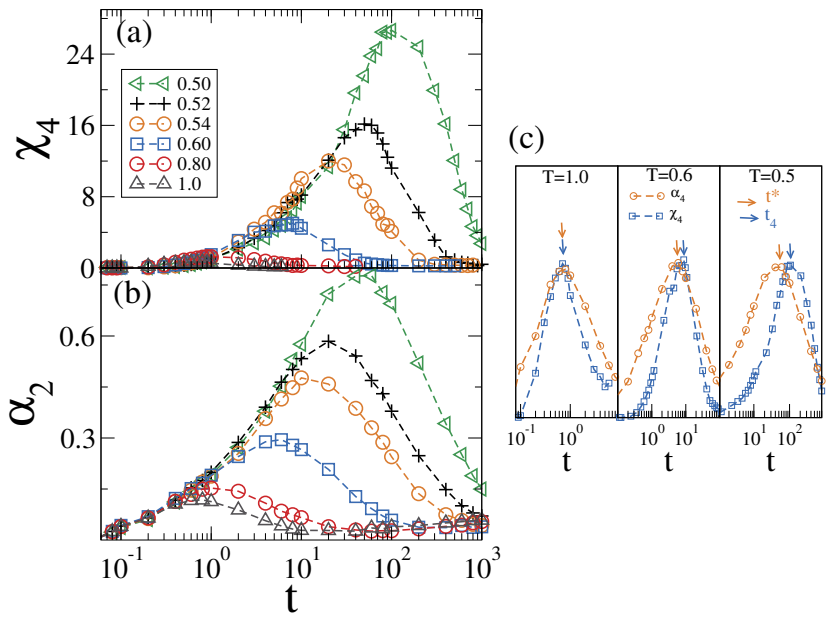

Figure 2. Two typical functions that define the dynamic heterogeneity and the characteristic times: a) the dynamic susceptibility $\chi_{4}(t)$ and (b) the non-Gaussian parameter $\alpha_{2}(t)$. c) The relative behavior of both functions to show the shifts that occur between the characteristic times $t^{*}$ and $t_{4}$, where its clear from it that for high temperatures these times are equal and at low temperatures these times differ.

relaxation time, rather than $\tau_{\alpha}$. On the other hand, $\tau_{\alpha}$ and $t_{4}$, are related with less mobile particles and therefore with the viscosity (from Maxwell's relation). Considering the simplified form of Stokes-Einstein relation through the form:

$\frac{D}{T} \propto \frac{1}{\tau_{\alpha}}$,

it can be evidenced that the temperature where breakdown of the Stokes-Einstein relation is manifested when the system loses the lineal relation between the two characteristic times: $t^{*}$ and $\tau_{\alpha}$ [46]. From figure $3 \mathrm{~b}$ the temperature at which the breakdown of SER is manifested (defined as $T_{S E B}$ ) can be derived. Comparing Figure 1 with Figure $3 \mathrm{~b}$, we note that the temperature $T_{A}$, where the activation energy begins to depend on temperature, is similar to $T_{S E B} \sim 0.7$. These results agree with other types of glass forming models, where $T_{A}$ and $T_{S E B}$ [7] are found to have similar values. However, this is not an universal observation, since there are other models where a similarity between $T_{S E B}$ and $\mathrm{Tc}$ of Mode-Coupling theory [6] is found.

In what follows, we describe a method to evidence a structural support to the dynamic behavior described above. The methodology is based on observing the dynamic susceptibility under the conditions of an isoconfigurational ensemble. In line with this, we propose to take the ensemble average $<>$ of the overlap between configurations at two different times in two different forms: it can be averaged considering several independent trajectories (the typical case); or by an ICE where all trajectories have the same a)
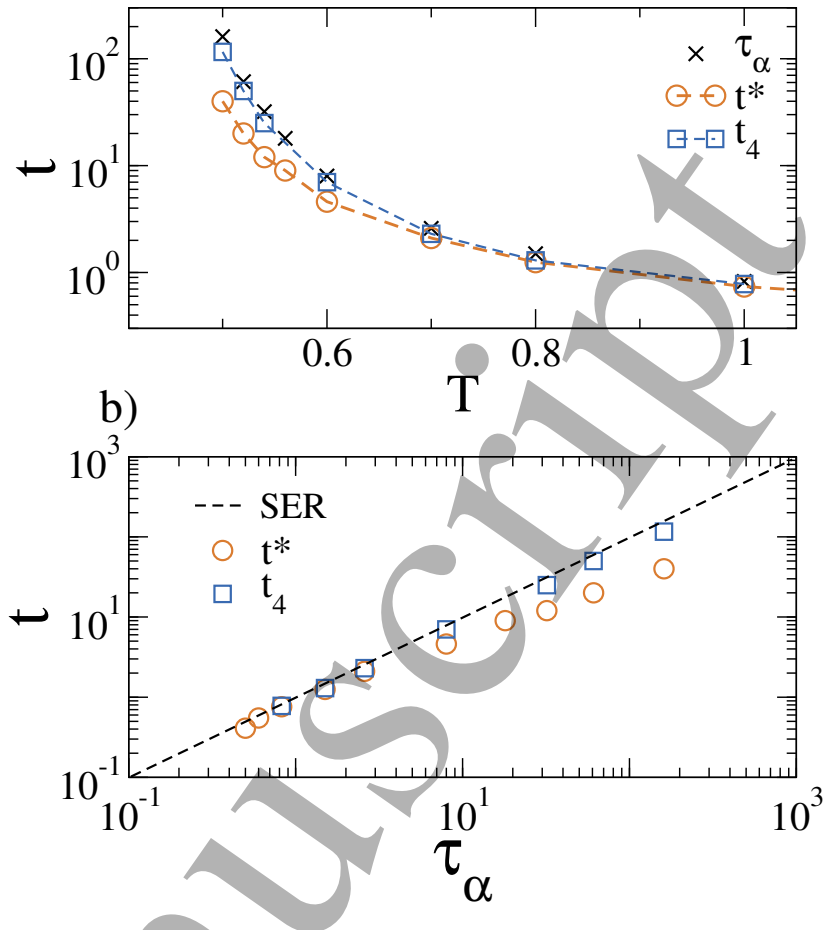

Figure 3. a) The different characteristic times of the system as a function of temperature. b) The characteristic times that describe the heterogeneous dynamics as a function of the structural relation time. It can be noted that $t_{4}$ presents a linearity with $\tau_{\alpha}$ for all temperature, while $t^{*}$ departs from the linearity at $T_{S E B} \sim 0.7$.

initial configuration. Figure 4 shows schematically the two possible situations that we use for the calculation of this magnitude. It is important to consider in both cases the same type of ensemble (e.g. canonical, microcanonical, etc.), because the value of dynamic susceptibility depends (slightly) on the type of ensemble used. [47, 48]. In our work, we used microcanonical ensemble for the two cases, so we are comparing dynamic fluctuations under the same type of statistical mechanics ensemble.

According to this,in the case $a$ we take the overlap function as:

$<Q(t)>=\frac{1}{N_{t r j}} \sum_{k}^{N_{t r j}} \sum_{i j}^{N} w\left(\left|r_{i}^{k}(0)-r_{j}^{k}(t)\right|\right)$

where $N_{t r j}$ represent the 500 different independent trajectories consider in the sum. It is a classical manner to quantify this magnitude[23].

In the case $b$ we consider overlap function as:

$<Q(t)>_{I C}=\frac{1}{N_{I C}} \sum_{k}^{N_{I C}} \sum_{i j}^{N} w\left(\left|r_{i}^{k}(0)-r_{j}^{k}(t)\right|\right)$

where $N_{I C}$ represent the 500 trajectories considered in the ICEM. In Figure 5 the behavior of the overlap

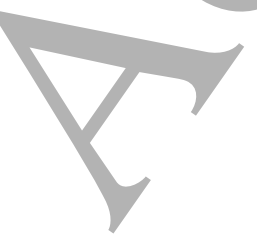


An alternative approach to evidence the structural conditioning in the dynamic slowdown in a polymer glass-former6

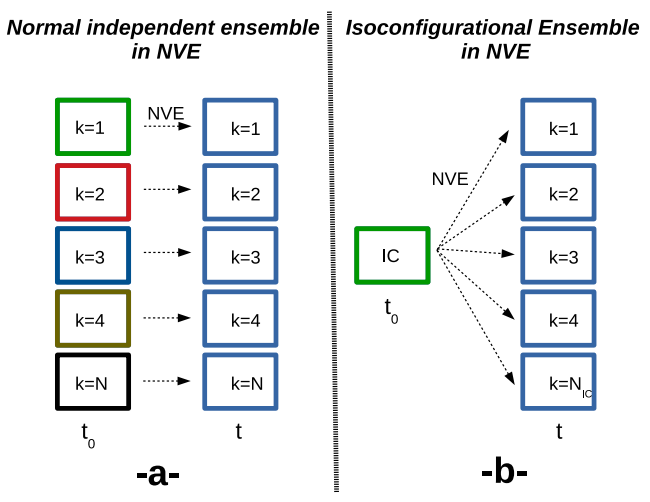

Figure 4. Scheme about the two ways to calculate the fluctuations in $Q(t)$ and the consequent $\chi_{4}(t)$. a) Through several independent configuration (case a). b) Within an ICE, several trajectories derived from the same initial configuration (case b).

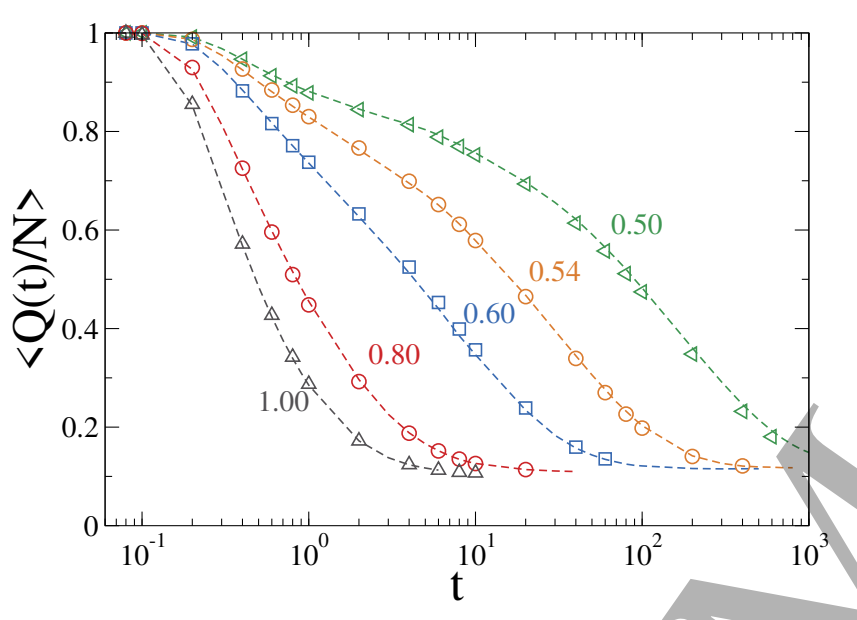

Figure 5. Average overlap function for the different temperatures calculated for the two cases of the figure: normal(dashed lines, case $a$ ) and ICE (symbols, case $b$ ).

functions is observed for both cases. It can be seen that there are no significant differences in the entire temperature range.

Then, we calculated $\chi_{4}(t)$ following equation 7 for the case $a$ (figure $2 \mathrm{a}$ ) and also the value of $\chi_{4-I C}(t)$ following the expression 8 for the case $b$ (figure $2 \mathrm{~b}$ ). At least 5 different independent ICE have been made at each temperature, each one we starting from different initial configurations. This is to ensure that the results do not depend on the initial configuration. The results at each temperature are similar.

Figure 6 shows the behavior of $\chi_{4}(t)$ for both cases over the entire temperature range. In both, heterogeneity grows when the temperature is lower and the time in which the largest heterogeneity occurs also grows. Moreover, the time in which the maximum is shown in the curves is the same for both cases. An

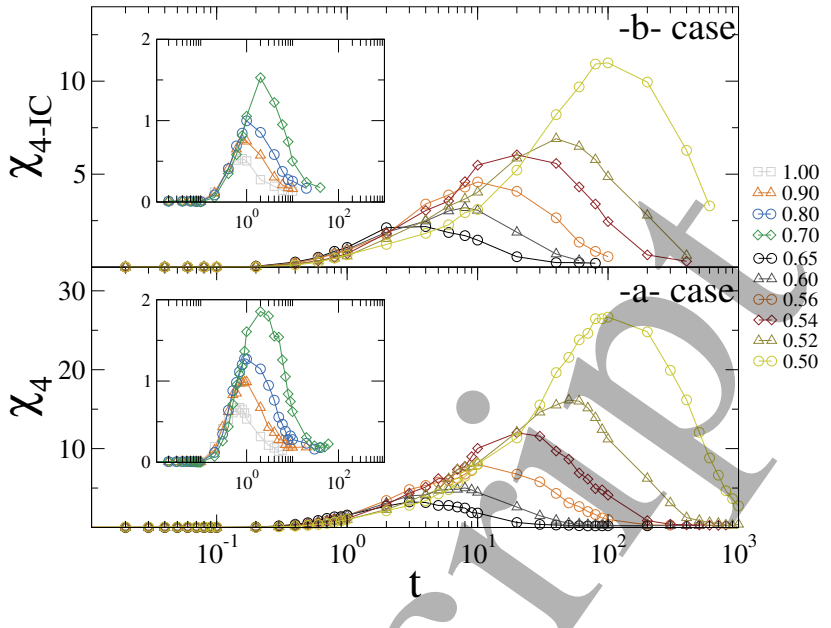

Figure 6. Behavior of $\chi_{4}(t)$ for the two cases at different temperatures. The inset contain the behavior for the highest temperatures.

interesting result from that figure is that $\chi_{4-I C}(t)$ has a lower value with respect to $\chi_{4}(t)$, which is more evident at low temperatures. We interpret this in the following way. Taking the equation 4 as the reference and following the case $a$ of the Figure 4, we can notice that the variability has two components, one due to the initial configurations and another component due to the dynamics of each trajectory. At high temperatures, where the structure does not influence dynamics, both cases are similar, because the variability in the initial velocities and the consequent differences in the mobilities is the main representative of the variability in $\chi_{4}$. On the other hand, at low temperatures, the structural component begins to be important, due to the fact that, in each initial configuration, there exists a structural heterogeneity that affects the mobilities of the particles at time scale of the order of $\tau_{\alpha}$. Then for case $a$ and at low temperatures the variability presents both components, the structural and the dynamic, and for this reason its value is higher than with respect to case $b$. Again, this result evidence that, in the supercooled system, there is a structural effect that affects dynamic behavior.

In order to check that these qualitative results of the comparative of $\chi_{4}\left(t_{4}\right)$ for case $a$ and case $b$ are independent from the initial condition in the ICE (the initial configuration that generate the ICEs), we have performed 300 independent isoconfigurational ensembles. This was done at two of the temperatures studied. In the figure 7 it can be observed that at the highest temperature, $\mathrm{T}=1.0$, the values of the different ICEs are similar to the average obtained for the case $a$. On the other hand, at $\mathrm{T}=0.6$, it is clear how $\chi_{4}\left(t_{4}\right)$ values are always lower than the average value of case a. For the others temperatures we at least 5 different 
An alternative approach to evidence the structural conditioning in the dynamic slowdown in a polymer glass-former 7

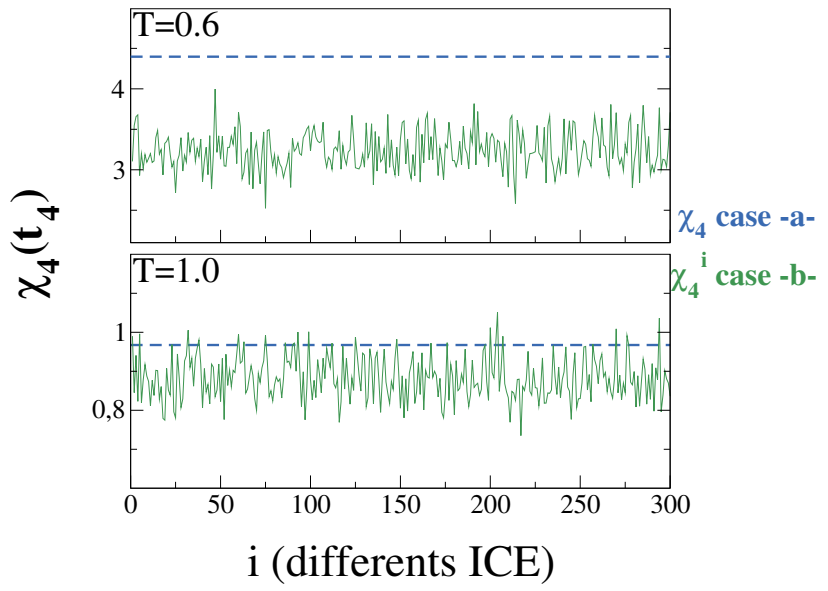

Figure 7. The maximum values that $\chi_{4}\left(t_{4}\right)$ acquires based on the different ICE realizations, where each $i$ corresponds to an ICE generated of 500 trajectories. The blue dashed lines correspond to the average obtained for case a taken from the 300 configurations that were used to generate the ICEs.

independent ICE have been made, each one starting from different initial configurations.

The peak value of dynamical susceptibility can be associated with a measure of the volume of space correlated during structural relaxation [26]. In our case, the value of the function at time $t_{4}$ (maximum value) is considered as a measure of the number of dynamic correlated particles $\left(N_{\text {corr }-D y n}\right)$ :

$N_{\text {corr-Dyn }}(T)=\max _{t}\left\{\chi_{4}(t, T)\right\}=\chi_{4}\left(t_{4}, T\right)$,

so we have two different values: the $N_{\text {corr-Dyn }}$ calculated for the case a, that is without ICE defined as $N_{\text {corr }-N}$; and for the case b, with the ICE defined as $N_{\text {corr }-I C}$. It is important to note that we are interested in the relative behavior of both cases, so that the absolute value of this magnitudes is not important at this point. The behavior for both cases is shown in Figure 8. It can be noticed that both curves practically overlap for high temperatures and at intermediate temperature, $T^{*}=0.7$, the curves begin to be easily discriminated. This can be better appreciated in the inset of Figure 8. At high têmperatures, when the structure does not have a marked influence, this ratio is approximately one, and for temperatures lower than $T^{*}$ this value begins to grow, indicating that as the glass former approaches the $T_{g}$, there is a larger effect of structure on the number of dynamically correlated particles measured by $\chi_{4}$. This allows the identification of onset of structure effects on dynamic behavior, as $T^{*}$.

Finally, this can be compared with the number of correlated particles defined from a purely structural or static correlation. To this end, the number of correlated particles calculated with the Point-to-Set (PtS) method is extracted from simulations performed

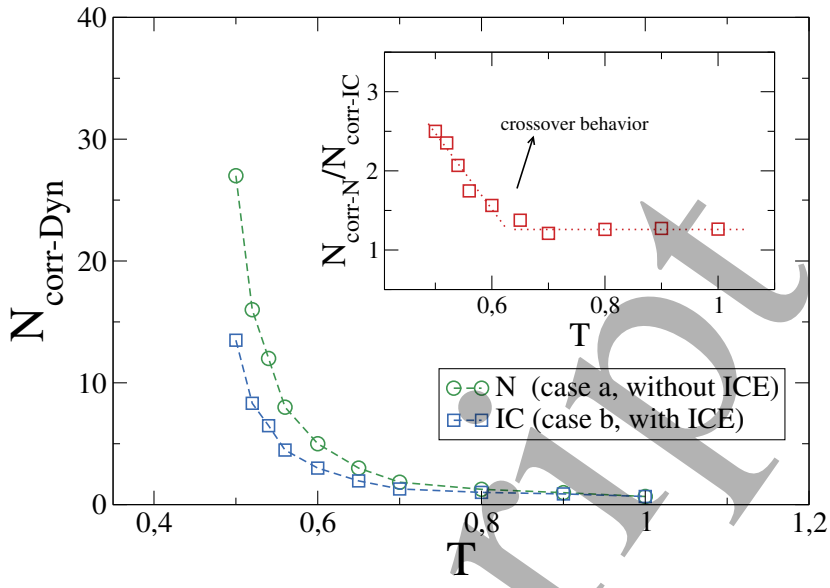

Figure 8. The number of dynamic/correlated particles calculated following expression 9 for both cases. The inset shows the relationship between them at the different temperatures.

in our previous work [39]. We calculate the number of correlated particles according to the PtS method by calculating: $N_{\text {corr }-P t S}(T)=4 / 3 \pi \rho(T)\left(\xi_{P t s}(T)\right)^{3}$, where $\xi_{P t s}(T)$ is the static correlation length of a spherical cluster from $\mathrm{PtS}$ method. In Figure 9, dynamic correlations $N_{\text {corr-Dyn }}$ (calculated from $\chi_{4}(T)$ for both cases are compared with $N_{\text {corr }-P t S}$ from PtS method. As usually observed, the number of dynamic correlations related to standard 4-point correlation functions, like $N_{c o r r-N}$, increase more abruptly with supercooling at low temperatures than the static correlations. On the other hand, $N_{\text {corr }-I C}$ displays a linear correlation with $N_{c o r r-P t S}$ in the whole range, this could mean that dynamic correlations reassured in the isoconfigurational ensemble are strongly coupled to static correlations in the whole temperature range.

As mentioned before, a central challenge in describing glass formation is the origin of the rapidly increasing relaxation time approaching $T_{g}$. This is the defining characteristic of fragile glass-forming fluids and it is related to dynamic heterogeneity. If we consider the temperature $T_{A}$, which marks the change in behavior that occurs in the relaxation of the system, where at $T>T_{A}$ the behavior is Arrhenius type, and for $T<T_{A}$ the system presents a non-Arrhenius behavior, we find that both $T^{*}$ and $T_{S E B}$ are close to $T_{A}$. From our results, we associate that this crossover behavior that happens at $T_{A}$, is due to a structural component emerging in the dynamic heterogeneity in the glass-former, which is not present at higher temperatures and manifest itself as a significant increase in correlations observed in a single trajectory, leading to a breakdown of both the Arrhenius dependence of relaxation time on temperature, and SER. On the other hand, when

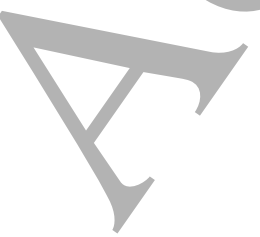




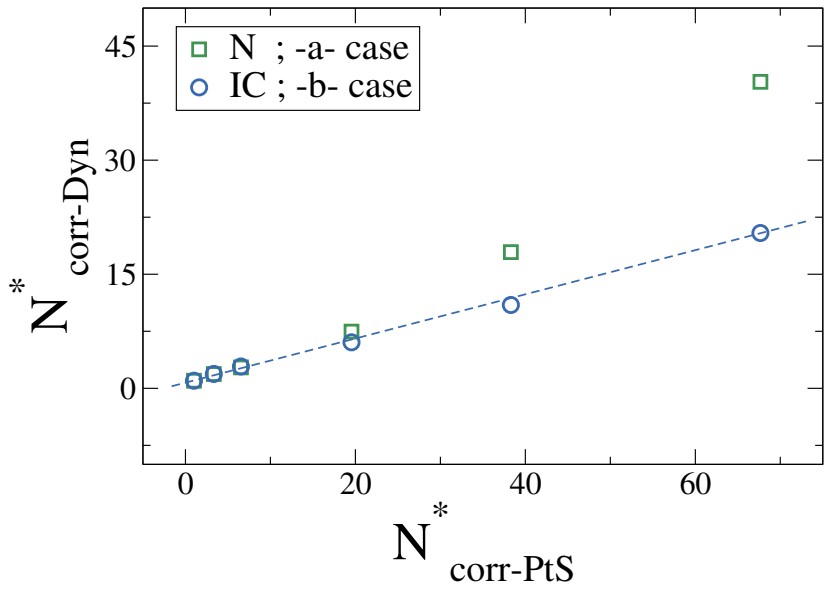

Figure 9. Number of dynamic correlated particles, $N_{\text {corr-Dyn }}$, calculated by the dynamic susceptibility for both cases (with and without ICE), in relation by the $N_{c o r r-P t S}$ calculated by the $\mathrm{PtS}$ method. All the magnitudes are related to the value of the highest temperature $(\mathrm{T}=1.0)$

dynamic correlations are measured in the ICE, a linear relationship with structural correlations is observed in the whole temperature range.

\section{Conclusion}

In this work, the relationship between non-Arrhenius behavior of relaxation, breakdown of SER and heterogeneous dynamic was analyzed, by means of MD simulations of a supercooled polymeric glassformer. Analyzing fluctuations in the dynamics of the system using an isoconfigurational ensemble at different temperatures, it was shown how the structure conditions the dynamics slowdown in the system below a certain temperature $T^{*}$. Our methodology is based in comparing the behavior of dynamic susceptibility under two different cases: on the one hand through a typical ensemble, and on the other by an IC ensemble, where in both cases the trajectories are generated following a microcanonical ensemble. We found that dynamic fluctuations for both cases are similar at high temperatures. However, for low temperatures the difference becomes evident, with a crossover behavior at $T^{*}$. This temperature is similar to $T_{A}$, the temperature at which super-Arrhenius behavior is observed, and to $T_{S E B}$, the temperature at which SER breaks down. This results reinforces the idea that this typical phenomenology, that characterize glassformers, is related to dynamic heterogeneity associated to structural effects.

Additionally, the number of correlated particles calculated from $\chi_{4}\left(t_{4}\right)$ in both cases was compared with a measure of structurally correlated particles, obtained from Point-to-set correlations. It was observed that the number of correlated particles measured in the ICE, $N_{\text {corr }-I C}$, displays a linear relationship with $N_{\text {corr }-P t S}$ from PtS in the whole temperature range, suggesting than a strong association between dynamic correlations in the ICE and static correlations may exist. On the other hand, the standard $N_{\text {corr }-N}$ from a typical ensemble deviates from this linear relationship at a temperature $T^{*}$. These results shows that this methodology puts in evidence that the structure conditions the dynamics slowdown in this glass-former and shed light in the apparent discrepancies between structural and dynamical length scales in supercooled liquids, reinforcing the thermodynamics views of glass transition. In addition, this methodology does not depend on the nature of the system and could be used to understand dynamic behavior and its connection with structure in different glass formers.

\section{Acknowledgments}

This work was supported by grants from Consejo Nacional de Investigaciones Científicas y Técnicas (CONICET, Argentina) and Universidad Nacional de Mar del Plata. CB and ERS are research fellows of CONICET. We used Mendieta Cluster from CCADUNC and Toko Cluster from FCEN-UNCuyo, which are part of SNCAD-MinCyT, Argentina.

\section{References}

[1] Cavagna A 2009 Physics Reports 47651 - 124 ISSN 03701573 URL http://www.sciencedirect.com/science/ article/pii/S0370157309001112

[2] Berthier L and Biroli G 2011 Rev. Mod. Phys. 83(2) 587-645 URL https://link.aps.org/doi/10. 1103/RevModPhys.83.587

[3] Royall C P and Williams S R 2015 Physics Reports 5601 - 75 ISSN 0370-1573 URL http://www.sciencedirect. com/science/article/pii/S0370157314004062

[4] Karmakar S, Dasgupta C and Sastry S 2016 Reports on Progress in Physics $\mathbf{7 9} 016601$ URL http://stacks. iop.org $/ 0034-4885 / 79 / i=1 / a=016601$

[5] Berthier L, Biroli G, Bouchaud J P and Tarjus G 2019 The Journal of Chemical Physics 150094501

[6] Pan S, Wu Z W, Wang W H, Li M Z and Xu L 2017 Scientific Reports $739938 \mathrm{EP}-$

[7] Sengupta S, Karmakar S, Dasgupta C and Sastry S 2013 The Journal of Chemical Physics 138 12A548

[8] Wei S, Evenson Z, Stolpe M, Lucas P and Angell C A 2018 Science Advances 4

[9] Kawasaki T and Kim K 2017 Science Advances 3

[10] Tarjus G and Kivelson D 1995 The Journal of Chemical Physics 103 3071-3073

[11] Kumar S K, Szamel G and Douglas J F 2006 The Journal of chemical physics $\mathbf{1 2 4} 214501$

[12] Han X, Li J and Schober H 2016 The Journal of chemical physics 144124505

[13] Adam G and Gibbs J H 1965 The Journal of Chemical Physics 43 139-146 URL https://doi.org/10.1063/1. 1696442

[14] Kirkpatrick T R, Thirumalai D and Wolynes P G 1989 
An alternative approach to evidence the structural conditioning in the dynamic slowdown in a polymer glass-former 9

Phys. Rev. A 40(2) 1045-1054 URL https://link.aps. org/doi/10.1103/PhysRevA.40.1045

[15] Biroli G, Bouchaud J P, Cavagna A, Grigera T S and Verrocchio P 2008 Nature Physics 4771 URL http: //dx.doi.org/10.1038/nphys 1050

[16] Patrick Royall C, Williams S R, Ohtsuka T and Tanaka H 2008 Nature Materials 7556 article

[17] Tanaka H, Kawasaki T, Shintani H and Watanabe K 2010 Nature Materials 9324 URL http://dx.doi.org/10. 1038/nmat2634

[18] Malins A, Eggers J, Royall C P, Williams S R and Tanaka H 2013 The Journal of Chemical Physics 138 12A535

[19] Liang Y C, Liu R S, Xie Q, Tian Z A, Mo Y F, Zhang H T, Liu H R, Hou Z Y, Zhou L L and Peng P 2017 Scientific Reports 743111 EP - article URL https: //doi.org/10.1038/srep43111

[20] Hallett J E, Turci F and Royall C P 2018 Nature Communications 93272

[21] Hocky G M, Coslovich D, Ikeda A and Reichman D R 2014 Phys. Rev. Lett. 113(15) 157801 URL https://link. aps.org/doi/10.1103/PhysRevLett.113.157801

[22] Lačević N, Starr F W, Schrøder T B, Novikov V N and Glotzer S C 2002 Phys. Rev. E 66(3) 030101 URL https: //link.aps.org/doi/10.1103/PhysRevE.66.030101

[23] Lačevic N, Starr F W, Schröder T B and Glotzer S C 2003 The Journal of Chemical Physics 119 7372-7387

[24] Berthier L, Biroli G, Bouchaud J P and Jack R L 2010 ArXiv e-prints arXiv:1009.4765

[25] Berthier L, Biroli G, Bouchaud J P, Cipelletti L, Masri D E, L'Hôte D, Ladieu F and Pierno M 2005 Science 310 1797-1800 ISSN 0036-8075 URL http://science. sciencemag.org/content/310/5755/1797

[26] Dalle-Ferrier C, Thibierge C, Alba-Simionesco C, Berthier L, Biroli G, Bouchaud J P, Ladieu F, L'Hôte D and Tarjus G 2007 Phys. Rev. E 76(4) 041510

[27] Charbonneau P and Tarjus G 2013 Phys. Rev. E 87(4) 042305 URL https://link.aps.org/doi/10. 1103/PhysRevE. 87.042305

[28] Kob W, Roldán-Vargas S and Berthier L $2011 \mathrm{Na}-$ ture Physics 8164 URL http://dx.doi.org/10.1038/ nphys 2133

[29] Flenner E and Szamel G 2012 Nature Physics 8696 EP - correspondence URL http://dx.doi .org/10.1038/ nphys2437

[30] Mei B, Lu Y, An L, Li H and Wang L 2017 Phys. Rev. E 95(5) 050601 URL https://link.aps.org/doi/10. 1103/PhysRevE.95.050601

[31] Hima Nagamanasa K, Gokhale S, Sood A K and Ganapathy R 2015 Nature Physics 11403

[32] Dunleavy A J, Wiesner K, Yamamoto R and Royall C P 2015 Nature Communications 66089 URL http://dx. doi.org/10.1038/ncomms7089

[33] Das R, Tah I and Karmakar S 2018 The Journal of Chemical Physics 149024501

[34] Tong H and Tanaka H 2018 Phys. Rev. X 8(1) 011041

[35] Widmer-Cooper A, Harrowell P and Fynewever H 2004 Phys. Rev. Lett. 93(13) 135701 URL https ://link.aps . org/doi/10.1103/PhysRevLett. 93.135701

[36] Widmer-Cooper A and Harrowell P 2006 Phys. Rev. Lett. 96(18) 185701 URL http://link.aps .org/doi/10. 1103/PhysRevLett.96.185701

[37] Widmer-Cooper A, Perry H, Harrowell P and Reichman D R 2008 Nature Physics 4711715

[38] Balbuena C, Gianetti M M and Soulé E R 2018 The Journal of Chemical Physics 149094506

[39] Balbuena C, Gianetti M M and Soul E R 2019 The Journal of Chemical Physics 150234508

[40] Grest G S and Kremer K 1986 Phys. Rev. A 33(5) 3628-3631 URL https://link.aps.org/doi/10.1103/ PhysRevA.33.3628
[41] Plimpton S 1995 Journal of Computational Physics 1171 - 19 ISSN 0021-9991 URL http://www.sciencedirect. com/science/article/pii/S002199918571039X

[42] Aichele M, Gebremichael Y, Starr F W, Baschnagel J and Glotzer S C 2003 The Journal of Chemical Physics 119 5290-5304

[43] Puosi F and Leporini D 2012 The Journal of Chemical Physics 136164901

[44] Puosi F, Chulkin O, Bernini S, Capaccioli S and Leporini D 2016 The Journal of Chemical Physics 145234904

[45] Yamazaki T 2014 The Journal of Physical Chemistry B 118 14687-14694

[46] Starr F W, Douglas J F and Sastry S 2013 The Journal of Chemical Physics 138 12A541

[47] Berthier L, Biroli G, Bouchaud J P, Kob W, Miyazaki K and Reichman D R 2007 The Journal of Chemical Physics 126184503 URL https://doi.org/10.1063/1.2721554

[48] Berthier L, Biroli G, Bouchaud J P, Kob W, Miyazaki K and Reichman D R 2007 The Journal of Chemical Physics 126184504 URL https://doi.org/10.1063/1.2721555 